\title{
Numerical experiments on the motion of the outer planets
}

\author{
GERALD D. QUINLAN \\ Canadian Institute for Theoretical Astrophysics, University of Toronto
}

July 1991

\begin{abstract}
.
We have integrated the motion of the four Jovian planets on Myr timescales in fictitious solar systems in which the orbits differ from those of the real solar system. A change of $\lesssim 1 \%$ in the major axis of any one of the planets from its real value can lead to chaotic motion with a Lyapunov exponent larger than $10^{-5} \mathrm{yr}^{-1}$. A survey of fifty solar systems with initial conditions chosen at random from a reasonable probability distribution shows the majority of them to be chaotic.
\end{abstract}

Key words: Celestial Mechanics - Chaotic Dynamics - Solar System

\section{Introduction}

In the past few years evidence has appeared suggesting that the orbits of the planets are chaotic. Sussman and Wisdom (1988) integrated the motion of the outer planets for $845 \mathrm{Myr}$ and found that Pluto's orbit is chaotic with a maximum Lyapunov exponent of $1 / 20 \mathrm{Myr}^{-1}$. Laskar (1989), using analytical averaging of the equations of motion to speed up the calculations, integrated the motion of all nine planets for $200 \mathrm{Myr}$ and found that the inner solar system is chaotic with a maximum Lyapunov exponent of $1 / 5 \mathrm{Myr}^{-1}$. Nobili et al. (1989) concluded from their integration of the outer planets that "the outer solar system over $100 \mathrm{Myr}$ shows dynamical features that are typical of non-regular regions of motion in the phase space of non-integrable dynamical systems." These results raise a number of questions.

First, how certain are we that the orbits are chaotic? Wisdom (this volume) has confirmed some of the results using his new mapping technique, and Laskar (1990) has identified the physical mechanism responsible for chaos in his integration (which has now been corroborated by Laskar et al. 1991), but the Lyapunov exponent of a nonlinear dynamical system can be a sensitive function of the position in phasespace, and hence our ignorance of the exact planetary masses and initial conditions will always leave us with some uncertainty (see, e.g., Milani et al. 1989).

Second, if the planetary orbits are chaotic, why do they look so regular? The integration of Sussman and Wisdom (1988) shows that the orbit of Pluto does not undergo any major changes over $845 \mathrm{Myr}$, approximately 40 times longer than the inverse Lyapunov exponent of $20 \mathrm{Myr}$. The inner solar system has presumably survived in roughly its present configuration for at least several Gyr, much longer than the inverse Lyapunov exponent of 5 Myr found by Laskar (1989). There is no evidence in the numerical integrations of any sudden changes in the orbits, e.g., in the eccentricity or major axis of one of the planets, as has been observed in integrations of the motion of asteroids located in chaotic regions of phase space (Wisdom 1987). We would like to know the probability of such changes occurring, as it does not appear possible to rule them out if the orbits are chaotic.

Finally, what role, if any, has chaos played in determining the general characteristics of our solar system? Are the planetary orbits any more or less chaotic than one would have expected? Is there a reason for this? 
In this paper we discuss some preliminary numerical experiments that address these questions. Our goal is not to study chaos in the real solar system, but rather to study a number of solar systems similar to the real solar system to determine how common chaos is, what the typical size of the maximum Lyapunov exponent is, and what the consequences of chaos are for the planetary orbits. To reduce the computational expense we have included only four planets, which are given the correct masses for the four Jovian planets (and also the correct order, i.e., the planet closest to the Sun has Jupiter's mass, the second closest has Saturn's mass, etc.). The Jovian planets contain most of the mass in the solar system and are only weakly perturbed by the other planets, so it is not a bad approximation to study them in isolation. If we find that chaos is common in solar systems with four planets then it can only be more common in systems with nine planets.

We have done two types of experiments. In the first we started the planets on the correct orbits for the Jovian planets of the real solar system, but with small changes to the initial major axes. In the second we started the planetary orbits from initial conditions chosen at random from a reasonable probability distribution. The integrations were followed for 1-5 Myr in the first type of experiment and for $1 \mathrm{Myr}$ in the second. We used the 13th-order Störmer multistep method with a stepsize of 36 days; the energy was usually conserved to better than one part in $10^{9}$.

To search for evidence of chaos we integrated the variational equations along with the equations of motion to follow the phase-space separation $d$ (determined from the positions and velocities of all the planets, measured in $\mathrm{AU}$ and $\mathrm{AU} / \mathrm{yr}$ ) between two nearby systems as a function of time. We then plotted

$$
\gamma(t)=\frac{1}{t} \log \left[\frac{d(t)}{d(0)}\right]
$$

versus $t$ on a log-log scale (see Benettin et al. 1976). For regular systems $\gamma$ decreases roughly as $1 / t$, but for chaotic systems, for which $d(t)$ grows exponentially with time, $\gamma$ eventually stops decreasing and approaches a constant (the maximum Lyapunov exponent). If the plot shows $\gamma$ decreasing as $1 / t$ until the end of the integration it can mean either that the system is regular or that the system is chaotic but that the chaos is too weak to be detected in the time interval considered. In the sections that follow we simply report the value of $\gamma$ at the end of the integration, but in each case where we say the integration was chaotic we have inspected the plot of $\gamma$ versus $t$ and have verified that $\gamma$ had stopped decreasing.

\section{Experiments on Solar Systems Close to the Real Solar System}

In these experiments we varied the initial major axis of the four Jovian planets, one at a time, by amounts ranging from $-10 \%$ to $+10 \%$. Figure 1 (a) shows the results obtained by varying the initial major axis of Uranus. In four of the seven integrations that appeared chaotic (the second, fourth, fifth, and sixth from the left) the chaos was clearly related to a low-order resonance between the mean motions of Uranus and another planet (the 2:5 and 1:3 resonances with Saturn, and the 2:1 resonance with Neptune), but in the other three integrations it was not obvious if the chaos resulted from one particular resonance. Figure $1(\mathrm{~b})$ shows the results from varying 

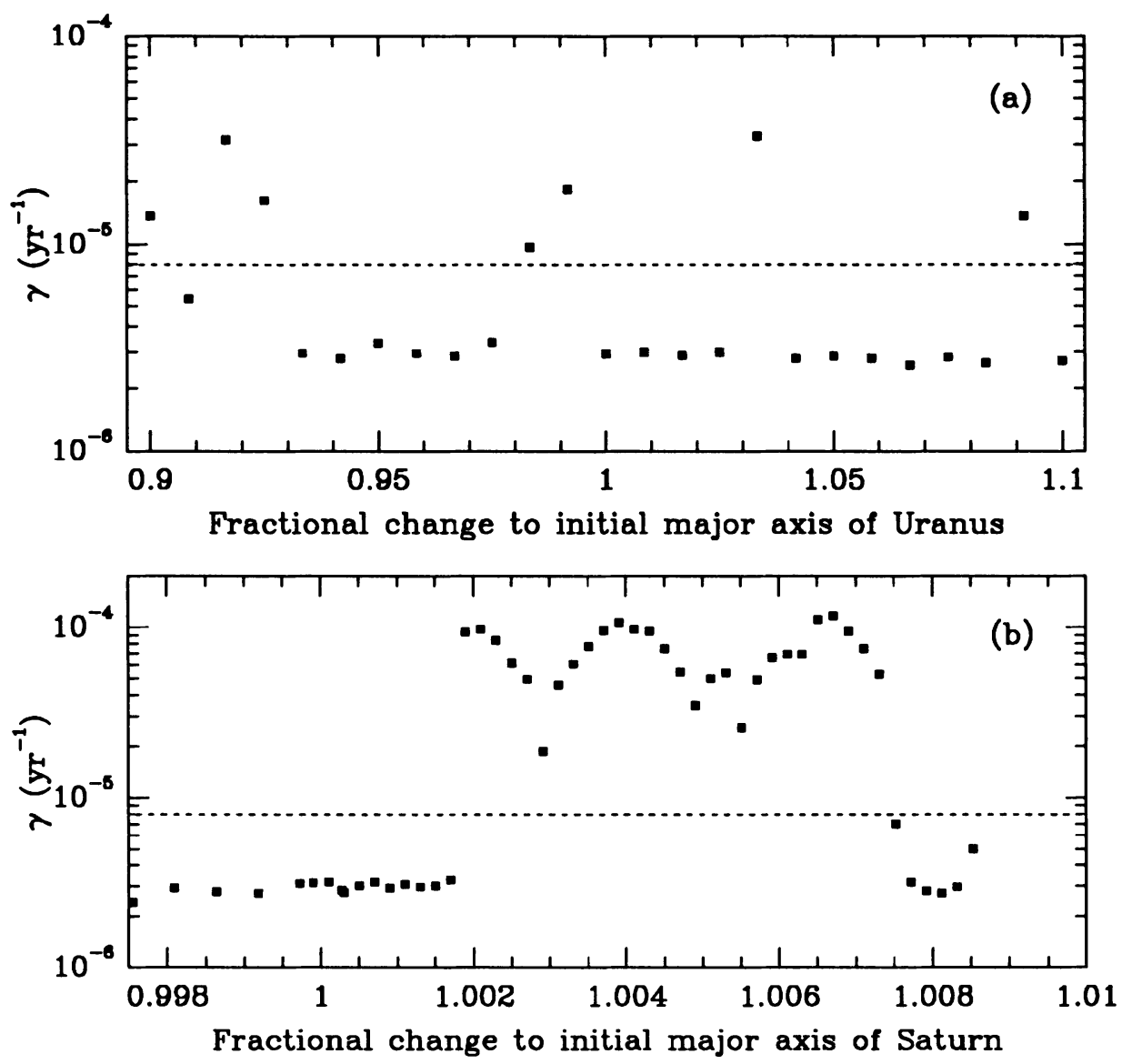

Fig. 1. Lyapunov exponents measured in $5 \mathrm{Myr}$ integrations of the outer planets in which the initial major axis of one planet differed from its real value by the indicated amount. The dashed lines separate the integrations that appeared chaotic (i.e., $\gamma$ had stopped decreasing with time) from those that did not. (a) Results from the four-planet JSUN system. (b) Results from the two-planet JS system.

the initial major axis of Saturn in a two-planet solar system containing only Jupiter and Saturn. The figure focuses on the chaotic zone near the 5:2 resonance between the mean motions of Jupiter and Saturn. Note that the Lyapunov exponent can be as large as $10^{-4} \mathrm{yr}^{-1}$. This figure looks similar if all four planets are included in the integration, but we have shown the results from a two-planet solar system to emphasize that this is sufficient to give chaos.

Similar results were obtained when the initial major axes of Jupiter and Neptune were varied: chaotic zones with Lyapunov exponents of $\gtrsim 10^{-5} \mathrm{yr}^{-1}$ were found in 
the vicinity of low-order resonances between the planetary mean motions. A change of order $1 \%$ or less in the major axis of any of the Jovian planets is enough to move the solar system into one of these chaotic zones. A number of these chaotic systems were integrated for times 100-500 longer than the inverse Lyapunov exponent. No signs of any "macroscopic instability" were observed (e.g., sudden changes in the major axes or eccentricities, orbits crossing or escaping), although the eccentricity of Uranus did sometimes get perturbed to values as large as 0.19 .

\section{Experiments on Randomly-Chosen Solar Systems}

We chose the initial orbital elements for these experiments in the following way. The three longitudes $\lambda, \omega$, and $\Omega$ were chosen at random between 0 and $2 \pi$. For the eccentricities and inclinations we chose $e^{2}$ and $i^{2}$ at random in the ranges $0-2 e_{\mathrm{rms}}^{2}$ and $0-2 i_{\mathrm{rms}}^{2}$, where the rms values are taken from the time averages of Applegate et al. (1986) for the Jovian planets: $e_{\mathrm{rms}}=0.04, i_{\mathrm{rms}}=0.086^{\circ}$. For the major axes we chose $\log (a)$ at random between $\log (4 \mathrm{AU})$ and $\log (35 \mathrm{AU})$. The choice of major axes was rejected if the planetary "feeding zones" overlapped, where the width $2 \Delta a$ of the feeding zone of a planet of mass $m$ is given by (Lissauer 1987)

$$
\frac{\Delta a}{a}=B\left(\frac{m}{3 M_{\odot}}\right)^{1 / 3}
$$

with $\mathrm{B}=3$. For example, if Jupiter is at $a_{J}=5.2 \mathrm{AU}$, Saturn must be at $a_{S}>7.6 \mathrm{AU}$. About $3 / 4$ of the choices of major axes were rejected by this criterion; the motivation for the criterion is that it seems unlikely that the planets would form so close together that their feeding zones overlap. For each integration we monitored the planetary orbital elements, the minimum separation between the planets, and the phase-space separation $d$ as a function of time. The results of fifty of these random solar systems are summarized in Figure 2. We divide the fifty systems into three groups.

In the first group are the eight highly chaotic systems whose final $\gamma$ values were $\gtrsim 10^{-3} \mathrm{yr}^{-1}$. These systems are what we might loosely refer to as macroscopically unstable. In four of the systems the ordering of the major axes switched during the integration, and this would likely have happened in the other four if the integrations had been continued (these highly chaotic integrations were stopped after times ranging from $10^{4}-10^{5} \mathrm{yr}$ because they were rapidly becoming inaccurate). Figure 2(a) shows that in all eight systems there was a close encounter between the planets (within $<1 \mathrm{AU}$ in six of the eight cases), but Figure 2(b) shows that it would have been difficult to predict this from the initial conditions. While these eight systems were clearly chaotic, the precise Lyapunov exponents are uncertain because $\gamma$ had not converged to a limit by the end of the integrations.

In the second group are the twenty-four systems that were chaotic but that did not exhibit the type of macroscopic instability seen in the previous group. The systems in the second group were all integrated for $1 \mathrm{Myr}, 20-400$ times longer than the inverse Lyapunov exponent, but no major changes in the orbits were observed: the eccentricities and inclinations remained small, the major axes did not change by much, and the planets remained well separated. 

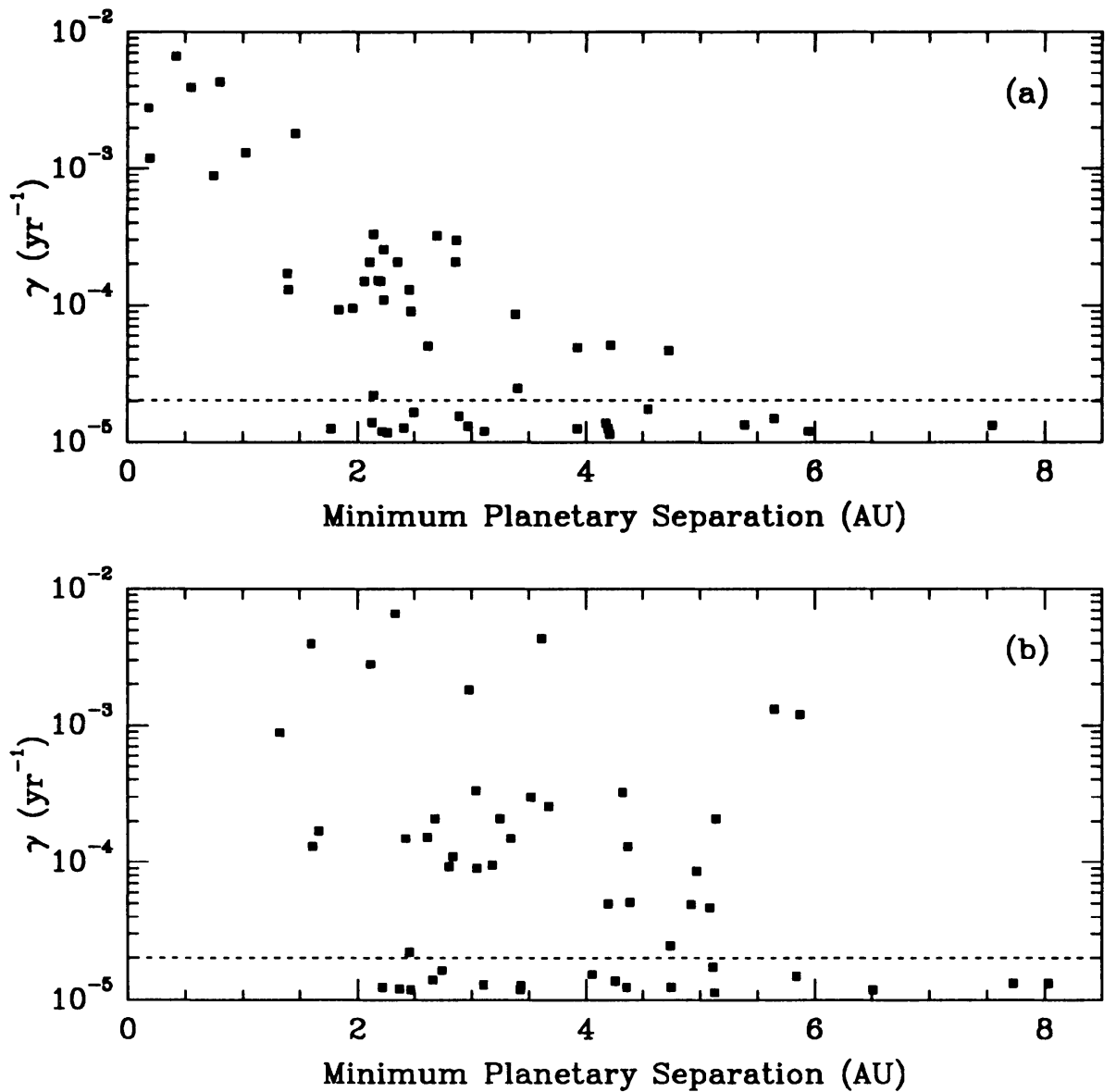

Fig. 2. Lyapunov exponents measured in $1 \mathrm{Myr}$ integrations of fifty randomly-chosen solar systems. The dashed lines separate the integrations that appeared chaotic (i.e., $\gamma$ had stopped decreasing with time) from those that did not. The abscissa gives the minimum planetary separation (a) measured during the integration and (b) computed on the assumption that the planetary orbital elements (other than the longitude $\lambda$ ) remain fixed at their initial values. The real JSUN planetary system (with the present-day orbital elements as the initial values) would be at abscissa $3.7 \mathrm{AU}$ in (a) and 4.2 $\mathrm{AU}$ in (b).

In the final group are the eighteen systems that appeared perfectly regular over the 1 Myr integrations, with no signs of chaos.

Figure 2(a) shows, at least for the chaotic systems, a correlation between the minimum planetary separation measured during the integration and the final value of $\gamma$. But Figure 2(b) shows the correlation to be much weaker if we predict the minimum planetary separation from the elliptical orbits on which the planets were initially placed. Note in Figure 2(b) that ten of the chaotic systems started with 
the planets on orbits that appeared to be more widely separated than are the orbits of the Jovian planets in the real solar system.

We have done some additional experiments which are not included in Figure 2. We integrated twenty-five systems where the orbits started with $e=i=0$. These proved to be much less chaotic than the fifty systems described above: twenty-one of the twenty-five appeared regular over a $1 \mathrm{Myr}$ integration, the other four were chaotic with $\gamma$ in the range $10^{-4}-10^{-3} \mathrm{yr}^{-1}$. We also integrated twenty systems in which the orbits were initially close enough that the feeding zones would overlap according to equation (2) with $B=3$ but not with $B=2$. These systems proved to be much more chaotic than the fifty described above: twelve of them had final $\gamma$ values $>10^{-3} \mathrm{yr}^{-1}$ (these integrations were stopped before $1 \mathrm{Myr}$ had elapsed), six were chaotic with $\gamma$ in the range $10^{-5}-10^{-3} \mathrm{yr}^{-1}$, and two appeared regular.

\section{Summary}

We draw several conclusions from these experiments. First, chaos appears to be a common feature of planetary systems, even when the planets are started on orbits that are well separated. Second, it is somewhat surprising how close the real solar system is to chaotic zones with large Lyapunov exponents $\left(10^{-5}-10^{-4} \mathrm{yr}^{-1}\right)$, although the significance of this is not clear. Finally, it is not surprising that Pluto and the planets of the inner solar system have existed on rather stable orbits for times much longer than the inverse of their reported Lyapunov exponents, because our experiments suggest that this is the rule rather than the exception.

\section{Acknowledgements}

This research was supported by NSERC (Canada). It is a pleasure to thank Scott Tremaine for his encouragement and helpful advice.

\section{References}

Applegate, J. H., Douglas, M. R., Gürsel, Y., Sussman, G. J., and Wisdom, J. 1986, "The outer solar system for 200 million years", A stron. J. 92, 176-194.

Benettin, G., Galgani, L., and Strelcyn, J.-M., 1976, "Kolmogorov entropy and numerical experiments", Phys. Rev. A14, 2338-2345.

Laskar, J. 1989, "A numerical experiment on the chaotic behaviour of the solar system", Nature 338, 237-238.

Laskar, J. 1990, "The chaotic motion of the solar system: a numerical estimate of the size of the chaotic zones", Icarus 88, 266-291.

Laskar, J., Quinn, T., and Tremaine, S. 1991, "Confirmation of resonant structure", submitted to Icarus.

Lissauer, J. 1987, "Timescales for planetary accretion and the structure of the protoplanetary disk", Icarus 69, 249-265.

Milani, A., Nobili, A. M., and Carpino, M. 1989, "Dynamics of Pluto", Icarus 82, $200-217$.

Nobili, A. M., Milani, A., and Carpino, M. 1989, "Fundamental frequencies and small divisors in the orbits of the outer planets", Astron. Astrophys. 210, 313-336.

Sussman, G. J. and Wisdom, J. 1988, "Numerical evidence that the motion of Pluto is chaotic", Science 241, 433-437.

Wisdom, J. 1987, "Chaotic dynamics in the solar system", Icarus 72, 241-275. 


\section{Discussion}

K.Aksnes - How would the picture of Lyapunov stability change if you were to change the masses of the planets?

Cl.Froeschle - Coming back to fictitious solar systems, what do you think of the increase of the masses as far as the stability is concerned?

$J$.Wisdom - In response to the questions about what happens if the masses of the outer planets are enhanced, I show a plot of final $\log d$ versus mass enhancement factor for 100 different mass enhancements ranging from 1 to 30 . Each integration covers 1 million years and was performed with the new mapping method. The plot shows that the outer planets become strongly chaotic with very small mass enhancements (about 1.5). This is very interesting. Recall Nacozy's (1976) result that, in much shorter integrations (only 10,000 years), the outer planets became strongly chaotic with a mass enhancement of 30 . Perhaps longer integrations would show chaos for smaller mass enhancements.

G.Quinlan - In most of our numerical experiments, the four planets were given the correct masses for Jupiter, Saturn, Uranus and Neptune, and therefore we cannot say for certain how things would change if the masses were given some other values. We did try some experiments where we enhanced the masses of the planets by factors ranging from 1 to 10 . We wanted to study how the maximum Lyapunov exponents depended on the enhancement factors, but the relationship we found was complicated and difficult to interpret (this is evident in the plot that J. Wisdom has shown). It is difficult to separate effects of changing the masses from the effects of the other changes that occur at the same time (e.g., changes in the location of resonances).

J.Laskar - In your study, you show that taking an outer solar system in a random manner, you end up most often in a system which looks much more chaotic than our actual solar system. I would like to stress out that the meaning of unstable for our solar system is very different than for another solar system. There is a constraint in our solar system which is that it needs to allow the apparition and persistence of life, which may be excluded in slightly unstable systems because of strong climatic variations.

B.Buti - What is your definition of instability? Are you saying that the system could be unstable in a region which you define as highly chaotic? Is there any specific parameters which would differentiate stable and macro-unstable systems?

G.Quinlan - Unfortunately, different people mean different things when they use the words stable and unstable when describing the motions of the planets. Some people say that the planetary motions are unstable if they cannot be described by quasi-periodic functions (according to this definition all chaotic solar systems are unstable). Jacques Laskar has suggested a special definition for our solar system: he says that the system should be considered unstable if the variation in the Earth's major axis and eccentricity is too large to allow life to survive on Earth. We have used the words stability or macroscopic stability to describe solar systems where the planetary orbits remain well separated and have low eccentricities and inclinations for all time, and we have used the word macroscopic instability to describe solar 
systems where there are large (or drastic) changes in the planetary spacings, eccentricities or inclinations. This is not a precise definition.

J.Palis - Just a remark on the subject of stability: positive Lyapunov exponent does imply some degree of sensitivity with respect to initial conditions, but does not necessarily implies big instability or diffusion; indeed, a hyperbolic subsystem is persistent, dynamically stable. To guarantee big instability or diffusion, we need more data about the system.

S.F.Dermott - Torques may have existed in the early solar system that drove the planets into resonances. These resonances are expected to be unstable, but is there any evidence that the planets (e.g., Uranus and Neptune that are close to a $2: 1$ resonance) could migrate from initial resonant configurations to their present configurations?

G.Quinlan - This is an interesting question, but I don't know the answer right now.

P.Goldreich - The width in semi-major axis of a libration zone, such as the $5: 2$ resonance between Jupiter and Saturn, depends on the planets' eccentricities. The eccentricities vary as the result of chaotic secular resonances. The current configuration of the solar system has Jupiter and Saturn very close to, but outside, the $5: 2$ resonance. Perhaps the planets' eccentricities were larger in the past and the critical argument spent time in libration.

G.Quinlan - I don't think the secular variation of the eccentricities was large enough to push the $5: 2$ resonance into libration. 